\title{
APLICAÇ̃̃O DO MÉTODO FRAM PARA ANÁLISE DA BAIXA COBERTURA VACINAL DO HPV NO MUNICÍPIO DE SÃO JOSÉ DOS CAMPOS
}

\author{
Adriana Cardoso Roberto \\ Instituto Tecnológico de Aeronáutica (ITA) \\ Pça. Mal. Eduardo Gomes, no 50, 12228-900 - São José dos Campos - SP \\ adrianacardososjc@hotmail.com \\ Alvimar de Lucena Costa Junior \\ Instituto Tecnológico de Aeronáutica (ITA) \\ Pça. Mal. Eduardo Gomes, nº 50, 12228-900 - São José dos Campos - SP \\ alvimar.lucena@gmail.com \\ Prof. Dr. Moacyr Machado Cardoso Júnior \\ Instituto Tecnológico de Aeronáutica (ITA) \\ Pça. Mal. Eduardo Gomes, nº 50, 12228-900 - São José dos Campos - SP \\ alvimar.lucena@gmail.com
}

\section{RESUMO}

O Papiloma Vírus Humano (HPV) é umas das Infecções Sexualmente Transmissíveis (IST) mais comuns, sendo capaz de causar diversos tipos de câncer, entre eles, o de colo de útero, quarta posição de morte na população feminina. Frente ao fato, o Ministério da Saúde promove campanhas de vacinação entre os adolescentes, porém, a cobertura vacinal das campanhas no Brasil tem ficado bastante abaixo das metas ministeriais. O artigo propõe o uso do Funcional Resonance Analysis Method (FRAM) para identificar funções do sistema de vacinação que permitam melhoras em sua resiliência, e consequente, melhoria da cobertura vacinal.

Palavra-chave: Engenharia de Resiliência; Cobertura Vacinal; FRAM.

\begin{abstract}
Human Papilloma Virus (HPV) is one of the most common Sexually Transmitted Infections (STIs) and can cause several types of cancer, including cervical cancer, which ranks fourth in the female population. To counter such a threat, the Ministry of Health promotes vaccination campaigns among adolescents, but the vaccination coverage of campaigns in Brazil has been well below ministerial targets. The article proposes the use of the Functional Resonance Analysis Method (FRAM) to identify intersections of the vaccination system that allow improvements in its resilience, and consequently, improved vaccination coverage.
\end{abstract}

Keywords: Resilience Engineering; Vaccinal Coverage; FRAM. 


\section{Como Citar:}

CARDOSO, A.R.; LUCENA, A.C.J.; MACHADO, M.C.J. Aplicação do Método FRAM para Análise da Baixa Cobertura Vacinal do HPV no Município de São José dos Campos. In: SIMPÓSIO DE PESQUISA OPERACIONAL E LOGÍSTICA DA MARINHA, 19., 2019, Rio de Janeiro, RJ. Anais [...]. Rio de Janeiro: Centro de Análises de Sistemas Navais, 2019.

\section{INTRODUÇÃO}

No ano de 2014, o Ministério da Saúde (MS), por meio do Programa Nacional de Imunizações (PNI), implementou a vacina contra o Papiloma Vírus Humano (HPV) no calendário de vacinação para adolescentes, nas faixas etárias preconizadas, disponíveis no Sistema Único de Saúde (SUS).

O HPV é umas das Infecções Sexualmente Transmissíveis (IST) mais comuns, sendo capaz de causar diversos tipos de câncer, como colo útero, pênis, garganta, vulva, vagina, ânus, além das verrugas genitais, sendo que o câncer do colo de útero ocupa a quarta posição de morte na população feminina Dentre os mais de 200 tipos de HPV, os mais comuns são os do tipo 6 e 11, causadores das verrugas anogenitais e os tipos 16 e 18, considerados os percussores dos carcinomas genitais (BOGAZ, 2016; BRASIL, 2014; MACHADO, 2016).

A importância desta cobertura vacinal em adolescentes é que eles possam chegar à fase adulta com menor risco de contrair o vírus do HPV.

O HPV é transmitido por contato direto com uma pessoa infectada e a principal forma de transmissão é por via sexual, que inclui contato oral-genital, genital-genital ou mesmo manual-genital. O Ministério da Saúde (MS) estabelece como meta a cobertura vacinal de $85 \%$ dos adolescentes, incluindo meninos e meninas, na faixa etária preconizada, sendo contemplados com duas doses da vacina. Para o MS, a cobertura vacinal é calculada pelo resultado do número de doses aplicadas dividido pela população alvo, multiplicado por 100. Cobertura vacinal satisfatória, inclui $85 \%$ de adolescentes vacinados com a primeira dose e $85 \%$ de adolescentes vacinados com segunda dose. A eficácia da vacina será mantida se os adolescentes receberem as duas doses previstas no calendário vacinal, a segunda dose, seis meses depois da primeira dose. Apesar dos investimentos na vacinação, a cobertura vacinal não apresenta uma linearidade entre as doses, de modo que existe uma grande diferença nas coberturas vacinais da primeira dose para a segunda dose. As Tabelas 1 e 2 a seguir mostram os dados vacinais no cenário nacional e no cenário do município de São José dos Campos (SP) no ano de 2016, caracterizando a problemática que envolve o sistema.

Tabela 1 - Número de primeiras doses (D1) e segunda doses (D2) de Vacina HPV realizadas em adolescentes de 9 a 13 anos no Brasil no ano de 2016.

\begin{tabular}{cccc}
\hline Doses / Idade & População & Dose & Cobertura \\
\hline D1 $(9$ anos $)$ & 1.590 .639 & 717.498 & $45,11 \%$ \\
D1 $(10$ anos $)$ & 1.645 .230 & 220.136 & $13,38 \%$ \\
D1 $(11$ anos $)$ & 1.705 .705 & 131.360 & $7,7 \%$ \\
D1 $(12$ anos $)$ & 1.745 .937 & 95.885 & $5,49 \%$ \\
D1 $(13$ anos $)$ & 1.752 .909 & 49.086 & $2,8 \%$ \\
D2 $(9$ anos) & 1.590 .623 & 252.301 & $15,86 \%$ \\
D2 $(10$ anos $)$ & 1.645 .218 & 283.543 & $17,23 \%$ \\
D2 $(11$ anos $)$ & 1.705 .730 & 222.491 & $13,04 \%$ \\
\hline
\end{tabular}




$\begin{array}{lccc}\text { D2 }(12 \text { anos }) & 1.745 .950 & 176.542 & 10,11 \% \\ \text { D2 }(13 \text { anos }) & 1.752 .921 & 87.434 & 4,99 \%\end{array}$

Fonte: Departamento de Informática do Sistema Único de Saúde (DATASUS) - Sistema de Informações do Programa Nacional de Imunizações (SIPNI).

Tabela 2 - Número de primeiras doses (D1) e segunda doses (D2) de Vacina HPV realizadas em adolescentes de 9 a 13 anos no município de São José dos Campos (SP), no ano de 2016.

\begin{tabular}{cccc}
\hline Doses / Idade & População & Dose & Cobertura \\
\hline D1 (9 anos) & 4.653 & 1.574 & $33,83 \%$ \\
D1 $(10$ anos $)$ & 4.854 & 464 & $9,56 \%$ \\
D1 (11 anos) & 5.087 & 315 & $6,19 \%$ \\
D1 $(12$ anos $)$ & 5.247 & 277 & $5,28 \%$ \\
D1 $(13$ anos $)$ & 5.286 & 98 & $1,85 \%$ \\
D2 $(9$ anos) & 4.653 & 577 & $12,4 \%$ \\
D2 $(10$ anos $)$ & 4.854 & 564 & $11,62 \%$ \\
D2 $(11$ anos $)$ & 5.087 & 456 & $8,96 \%$ \\
D2 $(12$ anos $)$ & 5.247 & 393 & $7,49 \%$ \\
D2 $(13$ anos) & 5.286 & 136 & $2,57 \%$ \\
\hline
\end{tabular}

Fonte: Departamento de Informática do Sistema Único de Saúde (DATASUS) - Sistema de Informações do Programa Nacional de Imunizações (SI-PNI).

Conforme mostrado na Figura 1, a cobertura vacinal Nacional se encontra abaixo da meta de $85 \%$ em todas as faixas de idade. Porém, quando se verifica a cobertura vacinal no município de São José dos Campos (SP), esta é ainda menor, não chegando a 3\% no caso da primeira dose para indivíduos na faixa dos treze anos de idade.

Figura 1 - Cobertura Vacinal pelo método do Ministério da Saúde.

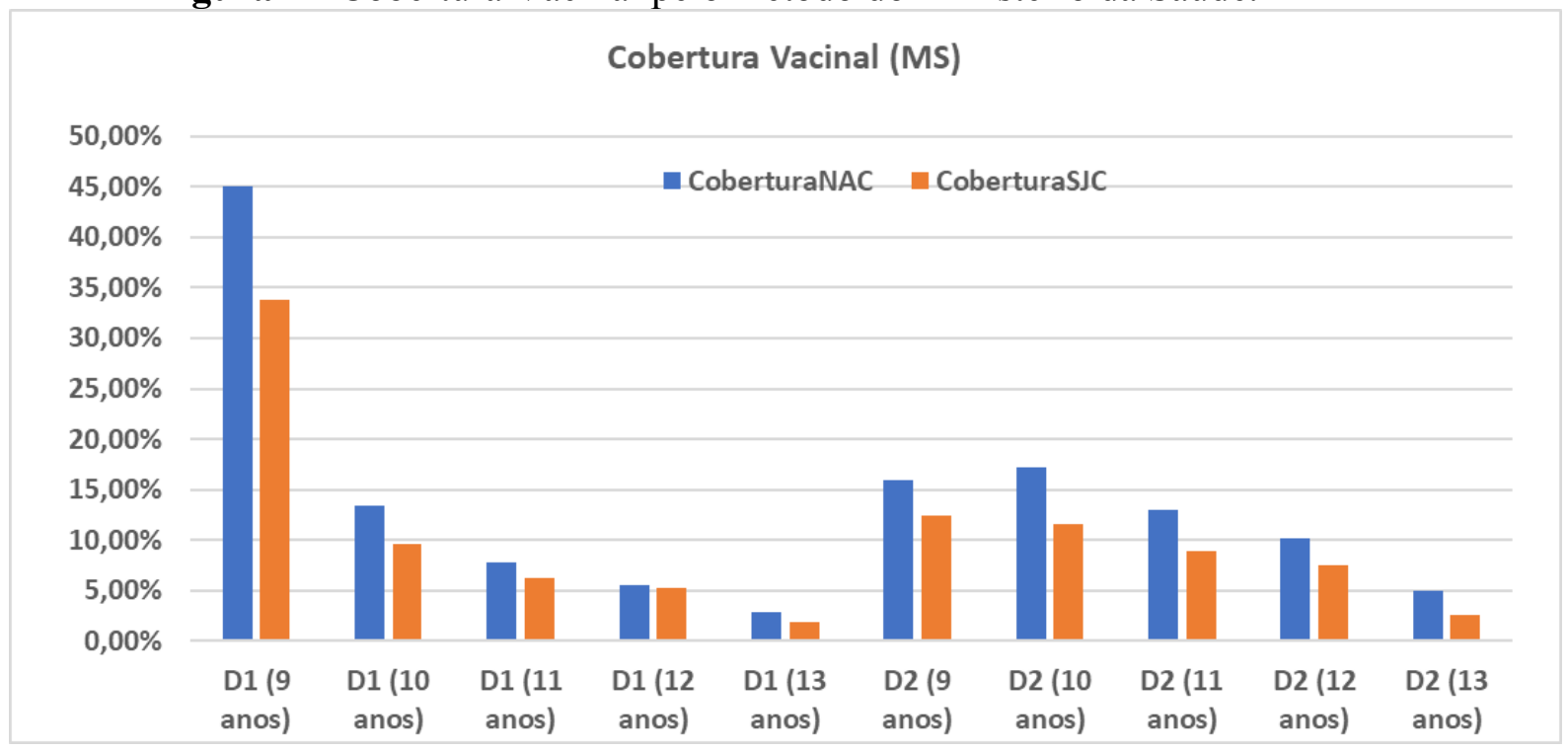

Fonte: Autores

A proposta deste trabalho é desenvolver um modelo de resiliência para a Campanha de Vacinação contra o HPV no município de São José dos Campos (SP), com o objetivo de alcançar a meta preconizada de $85 \%$ dos adolescentes vacinados nas primeiras doses e $85 \%$ dos adolescentes vacinados nas segundas doses.

Este trabalho está dividido em 5 seções. A primeira, introdutória, trata a 
problemática da campanha de vacinação do HPV no município de São José dos Campos (SP). Na sequência, a seção 2 apresenta o referencial teórico sobre Funcional Resonance Analysis Method (FRAM); a seção 3 apresenta a aplicação do Método na problemática do município; a seção 4 apresenta as propostas para a solução da problemática da baixa cobertura vacinal; a seção 5 relata as considerações finais, e por fim, a seção 6 sugere trabalhos futuros.

\section{REFERENCIAL TEÓRICO: FUNCTIONAL RESONANCE ANALYSIS METHOD (FRAM)}

O FRAM é um método que tem por objetivo entender como os sistemas realmente funcionam e como a variabilidade se propaga entre as suas funções, visando a desenvolver sistemas mais resilientes (CARVALHO, 2011).

Hollnagel (2006) define a resiliência como a capacidade intrínseca de um sistema para ajustar o seu funcionamento antes, durante, ou após alterações e perturbações, para que, mesmo após um grande acidente ou na presença de estresse contínuo, possa sustentar as operações necessárias.

Um sistema resiliente deve possuir três qualidades (antecipação, atenção e resposta) a fim de permanecer resiliente e sob controle. Isso se alinha com as quatro habilidades essenciais da resiliência propostas por Hollnagel et al. (2012) (HOLLNAGEL, WOODS, LEVESON, 2006):

- Saber o que fazer, envolve ações que visam responder às interrupções das operações previstas, através da implementação de um conjunto predefinido de respostas ou através de funções normais;

- Saber o que procurar, como controlar algo que é uma ameaça ou que tem potencial para tornar-se um problema. A observação deve incluir não apenas o que ocorre no ambiente, mas também o que ocorre no sistema em si (desempenho do sistema).

- Saber o que esperar, como antecipar as ameaças, as potenciais mudanças, os distúrbios, as pressões e principalmente as suas consequências;

- Saber o que aconteceu, como tirar lições úteis das experiências de sucesso e de fracasso.

Além disso, o método FRAM é elaborado e fundamentado em quatro princípios (HOLLNAGEL, 2012):

- Equivalência de sucesso e fracasso: tanto fracasso como sucesso são equivalentes, no sentido de que ambos têm a mesma origem. Uma outra forma de explicar este conceito é que atividades dão certo e errado pelos mesmos motivos. Ou seja, a variabilidade presente nos sistemas sócio-técnicos complexos reforça os atores envolvidos no seu gerenciamento a adaptar o sistema para mudanças associadas a constantes trocas de necessidades, recursos e cenários, porém tais as adaptações, às vezes, dão certo, às vezes, não dão certo;

- Ajustes aproximados: este princípio considera que a complexidade dos sistemas sócio-técnicos demanda uma série procedimentos com a finalidade de manter o desempenho nas mais variadas condições vivenciadas por estes sistemas. Estes procedimentos são incompletos e não abrangem todas as situações. Por este motivo, os ajustes são aproximados (não perfeitos) para corresponder à situação vivenciada;

- Princípio da emergência: a variabilidade combina-se de tal forma inesperada que ocorrem resultados inesperados e, às vezes, catastróficos. Por este motivo, é necessário buscar entender o sentido ou motivo por trás de algumas situações que promoveram um determinado assunto. Um evento não deve ser descrito como um "resultado", mas como uma 
"emergência" advinda dessas variabilidades do sistema;

- Princípio de ressonância funcional: como um fenômeno, a ressonância funcional descreve a variabilidade perceptível de um sistema sócio-técnico como resultado de vários ajustes aproximados que são básicos para as atividades profissionais diárias. Entre elas está o gerenciamento da variabilidade do sistema. Geralmente, a variabilidade ocasionada por apenas um componente do sistema ou por uma função isolada não é significativa a ponto de causar grandes impactos, porém, a variabilidade de diferentes funções pode combinar-se de maneira não prevista e provocar consequências graves (FOGAÇA, 2015). O termo ressonância aparece neste contexto com o sentido de salientar a propagação de efeito de uma função nas demais funções. Por exemplo, a variabilidade interna e externa de uma função entra em ressonância a variabilidade de outra função, a exemplo de uma frequência natural de uma estrutura, aumentando a amplitude da variabilidade e, consequentemente, seus efeitos.

A aplicação da metodologia FRAM consiste em quatro etapas podendo ser adaptadas de acordo com a necessidade (HOLLNAGEL, 2012):

Identificação e descrição das funções essenciais do sistema

Esta etapa consiste em identificar e descrever as funções ou atividades essenciais do sistema proposto. A identificação busca compreender as características de funcionamento normal de cada função da forma como é executada, e não como foi projetada ou imaginada". Para isto, o modelo FRAM utiliza seis aspectos que estão ilustrados na Figura 2: Input (entradas), Output (saídas), Preconditions (pré-condições), Resources (recursos), Time (tempo) e Control (controle). Estes aspectos permitem mapear como os recursos e as précondições estão relacionadas entre si, fornecendo uma melhor compreensão de como o trabalho está sendo realizado na prática. Este aumento da consciência situacional reflete positivamente na resiliência do sistema (FOGAÇA, 2015);

\section{Identificação da variabilidade}

Envolve caracterizar a variabilidade das diferentes funções identificadas, devendo endereçar tanto a variabilidade potencial como a real, estudando como tais variações podem se acoplar e derivar em resultados inesperados ou imprevistos, refletindo que estes impactos podem ser benéficos ou maléficos ao sistema. Ou seja, este passo se resume em observar o impacto de determinada característica em outra(s) característica(s) da mesma função ou de funções distintas. Isto ocorre porque a variabilidade de um aspecto pode afetar $\mathrm{o}$ desempenho de outro(s);

\section{Agregação da variabilidade}

Uma vez que a variabilidade de funções é identificada, devemos compreender como seus conjuntos influencia a forma como o sistema passa a funcionar. São determinadas instanciações, conjuntos de instâncias que descrevem os acoplamentos que existem ou podem existir em um determinado cenário ou conjunto de condições. Portanto, o foco resume-se em entender quais são as reações em cadeia da variabilidade de distintas funções, refletidas na estabilidade ou instabilidade do funcionamento do sistema como um todo;

\section{Desenvolvimento de recomendações}

É a busca de soluções possíveis para o gerenciamento de ocorrências de variabilidade de desempenho não controladas (ou ressonância funcional), podendo ocorrer de diversas maneiras. 
Figura 2 - Aspectos de cada função ou atividade do Functional Resonance Analysis Method.

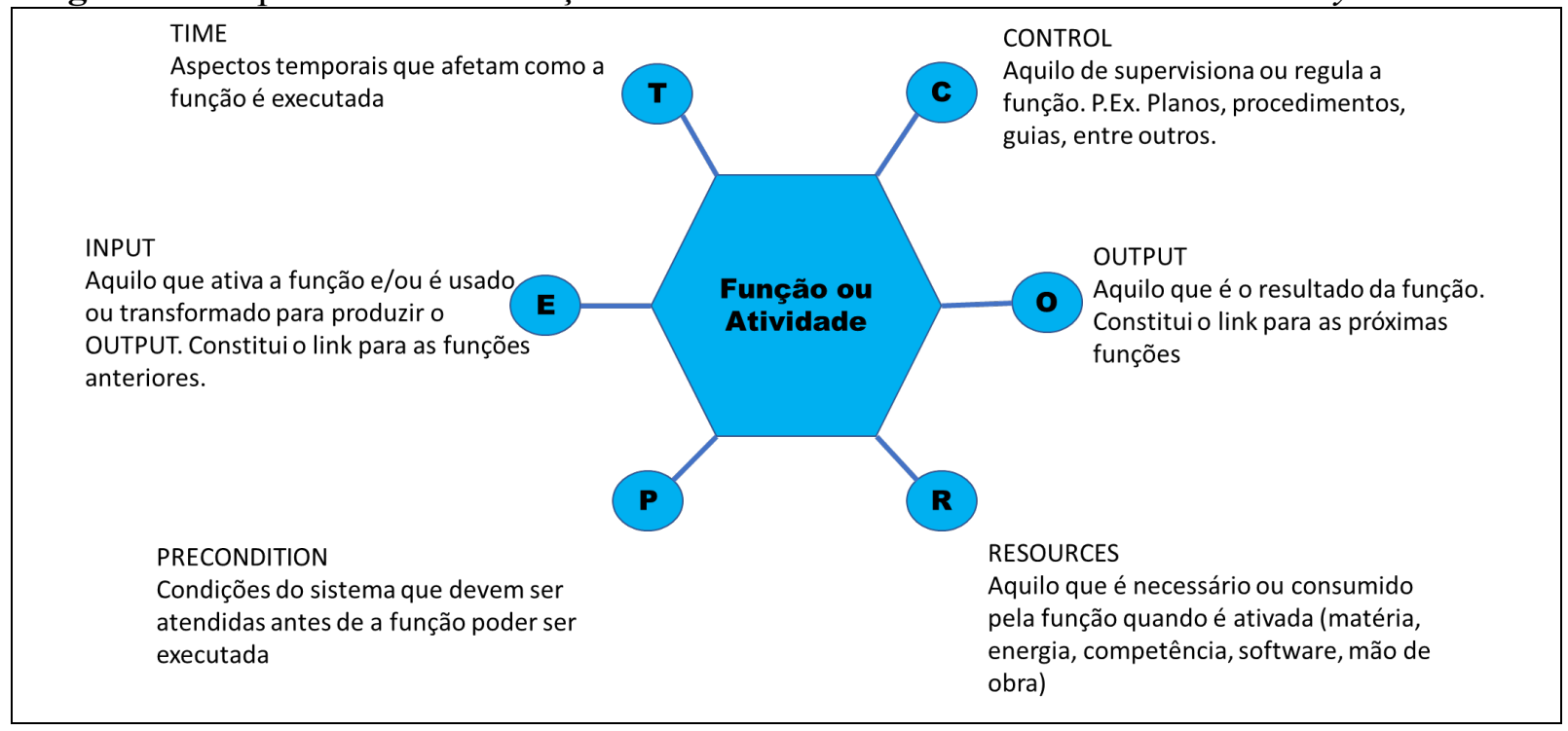

Fonte: adaptado de Hollnagel (2012).

Embora a modelagem FRAM seja relativamente nova, sua metodologia vem sendo aplicada com êxito para a compreensão e identificação da variabilidade e resiliência em sistemas complexos. O método FRAM não foi desenvolvido para aplicação específica no setor da saúde, porém demonstra diferentes abordagens para solução de problemas no setor da saúde (SOBEK e JIMMERSON, 2006).

O Setor da saúde se caracteriza pela sua complexidade, constante mudança e elevado número de envolvidos. Assim, requer métodos adequados, eficientes e eficazes, que evitem impactos desnecessários na segurança dos pacientes. Os serviços de saúde podem ser considerados sistemas complexos, pois possuem atributos de sistemas sócio-técnicos complexos, destacando-se o grande número de elementos interagindo dinamicamente, a diversidade de elementos, a variabilidade inesperada de suas funções e a resiliência do sistema como um todo (HEALY e BRAITHWAITE, 2006; MAGRABI et al., 2007; SUJAN et al., 2015; SAURIN e SOSA, 2013).

\section{MÉTODO}

Com a finalidade de viabilizar o estudo, foram conduzidas entrevistas com a enfermeira responsável pelo Programa de Imunização e a Chefia de Divisão da Vigilância Epidemiológica da Secretaria Municipal de Saúde do município. Essas entrevistas contribuíram para fornecer informações a respeito da organização hierárquica do Programa Nacional de Imunização, da estrutura física que o município possui para a aplicação da vacina, dos procedimentos para aplicação das vacinais, da conservação da vacina para manter sua eficácia, das estratégias necessárias para se iniciar uma Campanha de Vacinação e dos processos para desencadear ações para o cumprimento das metas de sucesso preconizada pelo Ministério da Saúde. Com base nestas informações, o problema foi estruturado, com a identificação dos agentes envolvidos (Stakeholders) e das dinâmicas socioculturais e de poder do contexto da situação problemática.

Entre os Stakeholders, destaca-se os adolescentes na faixa etária recomendada a vacinação, o responsável pelos adolescentes, a Secretaria Municipal de Saúde, os profissionais de saúde e os profissionais das escolas.

Entre as dinâmicas socioculturais do contexto da problemática, destaca-se as 
parcerias entre o Ministério da Saúde e Ministério da Educação para adesão à campanha de vacinação, com reforço em ambiente escolar e a confecção de material informativo sobre o HPV e suas consequências.

A Tabela 3 mostra os elementos das dinâmicas de Poder do contexto.

Tabela 3 - Dinâmicas de Poder.

\begin{tabular}{|c|c|}
\hline Órgão & Resumo da Dinâmica \\
\hline Ministério da Saúde & $\begin{array}{l}\text { Órgão do Poder Executivo Federal responsável pela organização } \\
\text { e elaboração de planos e políticas voltadas para a promoção, } \\
\text { prevenção e assistência à saúde }\end{array}$ \\
\hline Ministério da Educação & $\begin{array}{l}\text { Órgão da Administração Federal Direta, que tem como área de } \\
\text { competência a Política Nacional de Educação com poder de } \\
\text { elaborar estratégias dentro das escolas }\end{array}$ \\
\hline $\begin{array}{l}\text { Secretaria Municipal de } \\
\text { Saúde }\end{array}$ & $\begin{array}{l}\text { Órgão da Administração Municipal, responsável pela gestão das } \\
\text { políticas de saúde, com poder de planejar, controlar e organizar } \\
\text { estratégias para vacinação municipal }\end{array}$ \\
\hline $\begin{array}{l}\text { Unidades Básicas de } \\
\text { Saúde }\end{array}$ & $\begin{array}{l}\text { local prioritário para ações de saúde, com poder de aplicar a } \\
\text { vacina }\end{array}$ \\
\hline Escolas & $\begin{array}{l}\text { instituições concebidas para o ensino de alunos, com poder de } \\
\text { reunir o maior número de adolescentes e consequentemente, em } \\
\text { parceria com os profissionais de saúde realizar com segurança a } \\
\text { vacinação nos adolescentes }\end{array}$ \\
\hline
\end{tabular}

Fonte: Autores

No problema em questão, os tomadores de decisão são os especialistas em imunização e epidemiologia do município. A avaliação de vulnerabilidade e risco está tão entrelaçada com questões científicas que precisam ser realizadas por peritos científicos (AVEN, 2016).

Neste estudo, o FRAM é utilizado para uma análise retrospectiva da campanha vacinal contra o HPV realizada no município de São José dos Campos no ano de 2016, descrevendo o acoplamento entre a variabilidade de funções durante a campanha. Na análise foram identificadas e descritas as funções e a variabilidade potencial de cada aspecto, dividindo-os em humano, organizacional e tecnológico.

A Figura 3 demonstra a modelagem inicial da problemática do município. É possível identificar as funções representadas por hexágonos e seus aspectos a serem analisados estruturado por linhas e colunas, seguindo da esquerda para a direita, conforme o modelo atual do município. Os meios utilizados para atingir o sucesso nas duas etapas da vacinação foram identificados como funções do FRAM.

Cada função se conecta a outra via seu aspecto principal de interação. Por exemplo: a função "Armazenar em câmaras de vacinas ( $\left(1^{\text {a }}\right.$ dose $)$ " conecta-se via seu OUTPUT como o INPUT da função "Aplicar a primeira dose". Outro exemplo importante é o OUTPUT de "Criar parcerias com escolas" conectando-se como uma PRECONDITION para "Aplicar primeira dose" e também para "Executar palestras com profissionais da saúde". 
Figura 3 - Esquema inicial de encadeamento de atividades demonstrando pressões não descobertas (em vermelho), tendo a necessidade de análise aprofundada.

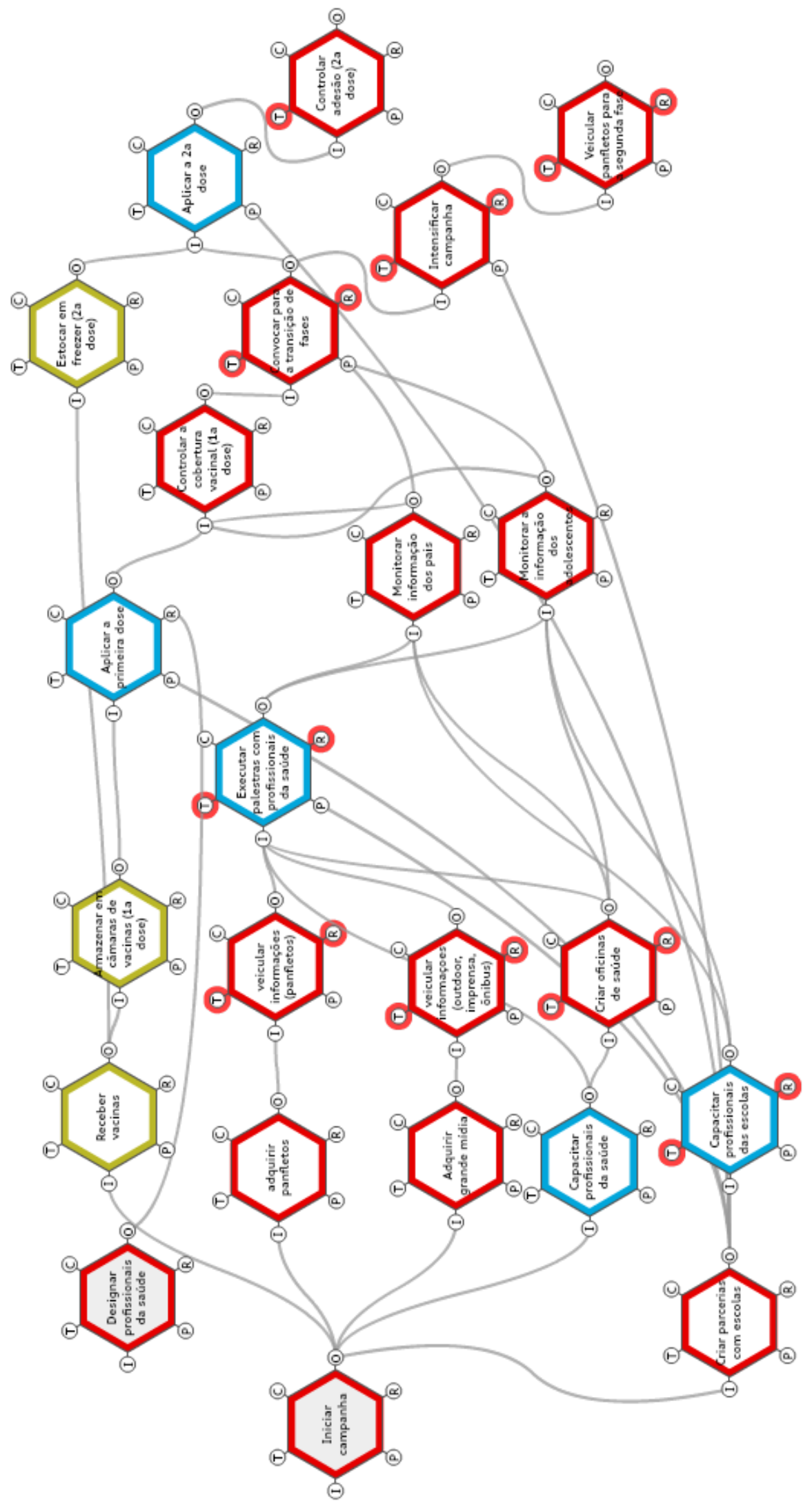

Fonte: Autores 
PRECONDITION e INPUT se repetem na função "Aplicar a segunda dose da vacina", destacando-se um aumento das funções. Pode-se também identificar, entre outros, o funcionamento tecnológico "estocar as vacinas em câmaras" para evitar perdas; o funcionamento organizacional "convocar para a segunda dose da vacina", "controlar a adesão da vacina na segunda dose", "controlar a cobertura vacinal da primeira dose", "convocar os adolescentes para a transição de fases", "intensificar a campanha", "veicular panfletos para a segunda fase da campanha"; e o funcionamento pessoal "continuar a capacitação de profissionais da saúde e escolar". A partir do entendimento da situação problemática e da estruturação do problema com base nas entrevistas com os tomadores de decisão foi possível desenvolver uma nova modelagem com ajustes do modelo existente e proposta para a solução do problema.

O "acidente" (evento indesejado, ou não atingimento da meta de cobertura vacinal) ocorre de acordo com a Figura 4, que é uma replicação da Figura 3, cuja instanciação do FRAM demonstra pelos "X" as falhas sequenciais. Restrições nos aspectos "tempo" e "custo" decorrem da decisão de executar a campanha, pressionando, por exemplo, a aquisição de panfletos e mídias de outdoor, sem um estudo prévio para determinar se é uma divulgação suficiente, eficiente ou eficaz para o atingimento da meta estabelecida. $O$ aspecto "tempo" também pressiona a função de "capacitação dos profissionais". Essas ressonâncias comprometem funções à frente da cadeia, como na execução de palestras, no número de profissionais capacitados e na assiduidade da população alvo. Sem a população conscientizada e assídua, a transição de fases é comprometida, prejudicando a aplicação do mínimo de doses necessárias para o cumprimento da meta.

Figura 4 - Sistema com as falhas posicionadas como "X" verde entre as funções.

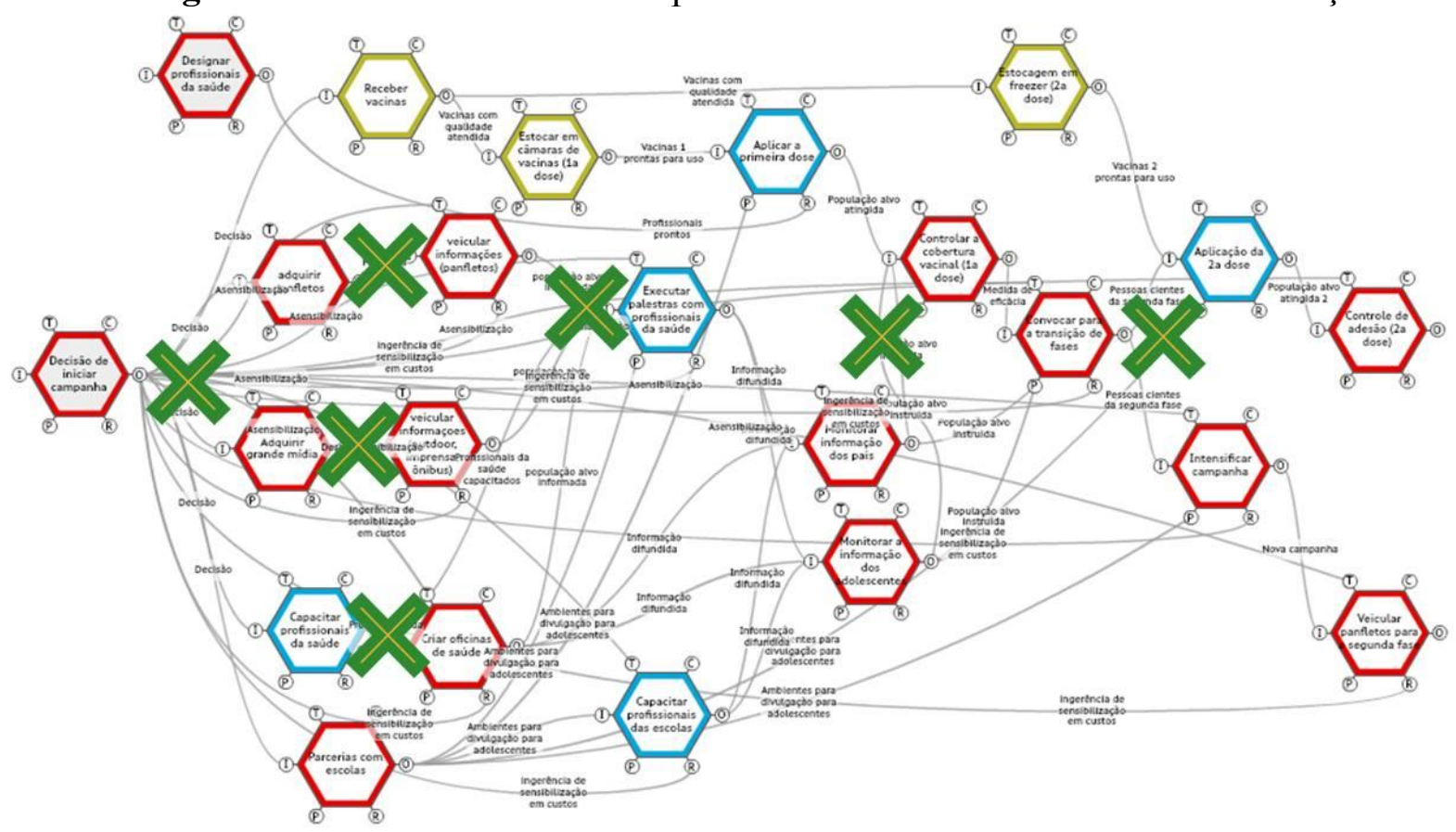

Fonte: Autores

\section{PROPOSTAS DE SOLUÇÃO PARA O PROBLEMA DE BAIXA COBERTURA}

Identifica-se que o modelo existente apresenta falhas por não explicar quais são as pressões que ressoam entre hexágonos (funções) de forma a não incentivar que o adolescente retorne para a administração da segunda dose da vacina, contribuindo para que a cobertura 
vacinal adequada seja atingida. Entre as falhas registradas, pode-se destacar a pressão por resultados, a falta de sensibilização do público alvo e a inexistência de cultura sobre a vacina indicada pelos profissionais de campo.

Por exemplo, detalhando-se o problema da pressão por resultados, vale informar que a vacinação não é obrigatória, e que a campanha é executada a partir de uma demanda formal do Ministério da Saúde, junto o estabelecimento de uma meta de $85 \%$ dos adolescentes vacinados. Assim, sob a ótica da gestão de risco, há uma pressão pela meta, refletindo em pressão por tempo e custo.

Buscando as falhas no sistema que impedem o sucesso da cobertura da segunda dose, destaca-se como exemplo a função "executar palestras com profissionais de saúde", que tem como finalidades alertar sobre os mitos existentes sobre a vacina e desanuviar os preconceitos da população, tendo como INPUT a função "capacitar os profissionais de saúde" sobre como sanar as dúvidas da população e como sensibilizá-la da importância da vacina. A função tem uma restrição temporal (aspecto TIME - T do esquema hexagonal) de um prazo limitado para que a informação seja difundida e suporte o atingimento da meta preconizada de vacinação. Assim como as restrições homem/hora dos profissionais de saúde e a verba destinada para a campanha, compreende-se que quanto mais profissionais de saúde capacitados por pessoa da população, melhor será a sensibilização e melhor a adesão a vacina. Dessa verificação, observa-se que a falta de sensibilização, ou a "dessensibilização", acontece por uma restrição de tempo e custo. A "dessensibilização" ressoa, contribuindo para o fracasso da campanha em atingir a meta num sistema resiliente. A Figura 5 exemplifica uma das funções identificadas para "executar palestra com profissionais da saúde" no Software FRAM model.

Figura 5 - Função "Executar palestra com profissionais da saúde".

\begin{tabular}{|l|l|}
\hline Nome da função & Executar palestras com profissionais da saúde \\
\hline Descrição & Palestras sobre mitos e preconceitos da vacina em comunidades \\
\hline Aspecto & Descrição do Aspecto \\
\hline \multirow{2}{*}{ Entradas } & Profissionais da saúde capacitados \\
\cline { 2 - 2 } & População alvo informada \\
\hline Saídas & Informação difundida \\
\hline Pré-requisitos & Ambientes para divulgação para adolescentes \\
\hline Recursos & Ingerência de sensibilização em custos \\
\hline Controle & \\
\hline Tempo & Dessensibilização \\
\hline
\end{tabular}

Fonte: Autores

Após a identificação do insucesso do sistema pelo esquema da Figura 3, cria-se um modelo demonstrando as pressões escondidas que precisam ser combatidas para aliviar o sistema complexo. É necessário desenvolver melhor as questões de sensibilização para a vacina e uma cultura na população, alvo e geral, para o entendimento da importância de vacinas e sua assiduidade, assim como completar o número de doses necessárias. A Figura 6 apresenta o novo modelo, com mais resiliência.

As propostas para combater os principais focos de ressonância de tempo e custo de forma atingir as metas são apresentadas a seguir, da função mais à esquerda para a mais à direita:

- Restringir acesso a outros serviços da unidade básica de saúde: Criar um processo para vacinação de forma que o paciente fique restrito a alguns processos no acesso ao uso da unidade básica de saúde, enquanto não passar na Sala de Vacina, para atualização da sua carteira de vacinal;

- Desenvolver cultura: Criar cultura de vacinação com a população 
independente de campanha, sensibilizando sobre os riscos de se desenvolver a doença, caso não seja vacinado, de forma contínua e perene;

- Monitorar a sensibilização: Função para mensurar o entendimento da população quanto a doença transmitida pelo Vírus do HPV e da necessidade da vacinação nos adolescentes como meio de proteção da doença;

- Obter feedback da conscientização: Após as palestras aplicar entrevistas ao público alvo para entender se a população compreendeu a necessidade da campanha e sua adesão, identificando pontos falhos da campanha, bem como identificar as pessoas que precisam de maior atenção;

- Criar mutirões em bairros com baixa adesão a vacina: usar a força militar juntamente com os profissionais da saúde, realizando o monitoramento das carteiras de vacinação de casa em casa, de forma a identificar diretamente as pessoas que estão com a vacinação atrasada;

- Ofertar vacinação: Ao identificar as pessoas com vacinação atrasada, durante o mutirão ofertar e, se possível, realizar a vacina no momento. Será necessário o encaminhamento do adolescente a unidade de saúde mais próxima juntamente com um profissional de saúde.

Trabalhar o processo de tomada de decisão dentro dos órgãos de saúde em todos os seus níveis hierárquicos, apontando ressonâncias que levam a decisões sob demanda que ressoarão no campo da vacinação também é uma providência importante.

\section{CONSIDERAÇÕES FINAIS}

O objetivo deste estudo foi desenvolver um modelo de resiliência para a Campanha de Vacinação contra o HPV no município de São José dos Campos (SP), com o objetivo de alcançar a meta preconizada de $85 \%$ dos adolescentes vacinados nas primeiras doses e $85 \%$ dos adolescentes vacinados nas segundas doses.

Os dados apresentados na Tabela 2 demonstram o insucesso na Campanha de Vacinação em São José dos Campos, onde não existe suficiente adesão na primeira dose (D1) ou na segunda dose (D2). Como não há adesão, não há resiliência no sistema.

A cultura é um fator fundamental para gerar a adesão à vacinação. A população sensibilizada quanto ao perigo da doença e orientada que a vacinação é um dos mecanismos para prevenção e aumentará gradativamente a adesão à vacinação. A falta de informação da população sobre a vacina contra o HPV, aumenta a falta e adesão para o retorno a segunda dose da vacina. Campanhas esporádicas, que geram as pressões de tempo e a subsequente ruína do sistema. O problema da baixa adesão está na própria qualidade do início da campanha, a forma como é decidido e executado, respondendo-se a uma percepção imediata, demanda, diferentemente de se buscar uma sensibilização perene sobre vacinas e especialmente do risco do HPV.

A proposta de modificação e inclusão de funções aumenta a resiliência do sistema, o que deve permitir uma melhor cobertura vacinal no município, possibilitando alcançar a meta numérica estabelecida e uma imunização eficiente da população. 
Figura 6 - Sistema da campanha de vacinação com as pressões de "dessensibilidade" ressoando pelas funções.

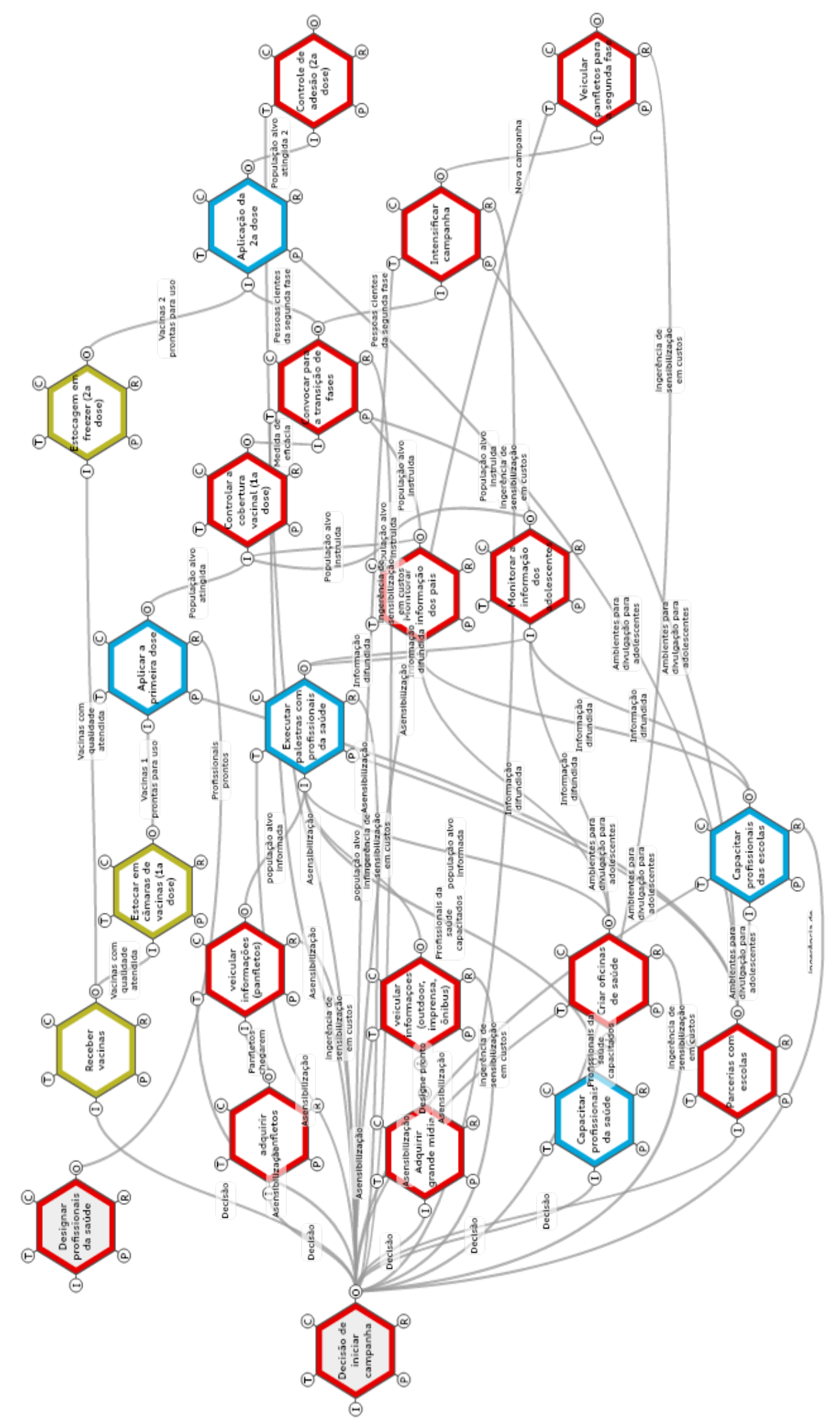

Fonte: Autores 


\section{TRABALHOS FUTUROS}

A abordagem do método FRAM enriqueceu o processo de entendimento da situação problemática, assim como a percepção de cada tomador de decisão. A participação do tomador de decisão foi fundamental para modelar o problema e identificar as falhas. No entanto, por ser um problema complexo, sugere-se que o modelo seja aplicado em setores censitários. Estas perspectivas ajudariam a identificar as falhas no sistema e propor melhorias de uma forma mais rápida.

\section{REFERÊNCIAS BIBLIOGRÁFICAS}

[1] AVEN, T. Risk assessment and risk management: Review of recent advances on their foundation. European Journal of Operational Research, Elsevier B.V., v. 253, n. 1, p. 113, 2016.

[2] BERTONI, V.; ROSSO, C.; SAURIN, T. (2017). Redes Sociais e Método de Análise de Ressonância Funcional em consonância ao Mapeamento do Fluxo de Valor em uma emergência hospitalar. VII Congresso Brasileiro de Engenharia de Produção.

[3] BOGAZ, C.; AMORIM, A.C. (2016) Meninos também serão vacinados contra HPV. Disponível em Acesso em: 09 fevereiro 2019.

[4] BRASIL. Informe Técnico sobre a vacina Papiloma Vírus (HPV) na atenção básica. Brasília (2014). Disponível em https://portalarquivos2.saude.gov.br/images/pdf/2015/junho/26/Informe-T--cnicoIntrodu----o-vacina-HPV-18-2-2014.pdf. Acesso em: 27 fevereiro 2019.

[5] CARVALHO, P.V. R. (2011). The use of Functional Resonance Analysis Method (FRAM) in a mid-air collision to understand some characteristics of the air traffic management system resilience. Reliability Engineering \& System Safety, v. 96, n. 11, p. 1482-1498. DOI: 10.1016/j.ress.2011.05.009

[6] FOGAÇA, L. B. (2015). Tomada de decisão e equilíbrio de metas conflitantes no gerenciamento de interrupções de voo em empresa de transporte aéreo regular. Dissertação (Mestrado em Administração e Negócios) - Faculdade de Administração, Contabilidade e Economia, Pontifícia Universidade Católica do Rio Grande do Sul, Porto Alegre.

[7] HEALY, J.; BRAITHWAITE, J. (2006). Designing safer health care through responsive regulation. Medical Journal of Australia, 184(10), S56 doi: 10.5694/j.1326-5377.2006.tb00364.x

[8] HOLLNAGEL, E. (2006). Resilience: the challenge of the unstable. In: Hollnahel, E.; Woods, D.D.; Leveson, N. (Ed.). Resilience Engineering: concepts and precepts. Aldershot, UK:Ashgate, 2006. p. 9-17.

[9] HOLLNAGEL, E.; SPEZIALI, J. (2008). SKI Report 2008:50 Study on Developments in Accident Investigation Methods: A Survey of the" State-of-theArt. ISSN 1104-1374. Disponível em https://inis.iaea.org/collection/NCLCollectionStore/_Public/40/012/40012565.pdf Acesso em 27 de setembro de 2019.

[10] HOLLNAGEL, E. (2008). The changing nature of risk. Ergonomics Australia Journal, 22(1-2), 33-46. 
[11] HOLLNAGEL, E; PARIÈS, M. J.; WOODS, D.; WREATHALL, J. (2011) Resilience engineering in practice: a guidebook. UK: Ash Gate Publishing. ISBN: 978-1-40941036-2 (ebk)

[12] HOLLNAGEL, E. (2012) FRAM: the functional resonance analysis method: modelling complex socio-technical systems. Farnham: Ashgate Publishing

[13] LEVESON, N. G. (2001). Evaluating Accident Models Using Recent Aerospace Accidents, Part 1: Event-Based Models. Disponível em http://sunnyday.mit.edu/nasa-class/nasa-report.pdf. Acesso em 27 de setembro de 2019.

[14] MACHADO, P. A. T. (2016). Cobertura Vacinal Contra o HPV em Meninas de 09 a 11 Anos no Município de Rolim de Moura - RO. Revista Enfermagem e Saúde Coletiva, v. 1, n. 2, p. 74-90, 2016, ISSN: 2448-394X, Faculdade São Paulo, 2016.

[15] MAGRABI, F.; MCDONNELL, G.; WESTBROOK, J. I.; COIERA, E. (2007). Using an accident model to design safe electronic medication management systems. In Medinfo 2007: Proceedings of the 12th World Congress on Health (Medical) Informatics; Building Sustainable Health Systems (p. 948). IOS Press.

[16] SAURIN, T.; SOSA, S. (2013). Assessing the compatibility of the management of standardized procedures with the complexity of a sociotechnical system: Case study of a control room in an oil refinery. Applied ergonomics, Vol. 44, n. 5, p. 811823, 2013. https://doi.org/10.1016/j.apergo.2013.02.003

[17] SOBEK, D. K.; JIMMERSON, C. (2006). A3 Reports: Tool for Organizational Transformation. IIE Annual Conference. Proceedings. Institute of Industrial Engineers-Publisher, p. 1.

[18] SUJAN, M.; SPURGEON, P.; COOKE, M.; WEALE, A.; DEBENHAM, P.; CROSS, S. (2015). The development of safety cases for healthcare services: practical experiences, opportunities and challenges. Reliability Engineering \& System Safety, Vol. 140, p. 200-207, 2015. 